\title{
Mast cells-derived MiR-223 destroys intestinal barrier function by inhibition of CLDN8 expression in intestinal epithelial cells
}

\author{
Musheng $\mathrm{Li}^{1 \dagger}$, Junhong Zhao ${ }^{1 \dagger}$, Meiwan Cao ${ }^{1 \dagger}$, Ruitao Liu', Guanhua Chen ${ }^{1}$, Songyu Li ${ }^{2}$, Yuanwen Xie', \\ Jing Xie ${ }^{1}$, Yang Cheng ${ }^{1}$, Ling Huang ${ }^{1}$, Mingmin Su${ }^{4}$, Yuxin Xu ${ }^{5}$, Mingyue Zheng ${ }^{6}$, Kejian Zou ${ }^{7}$, Lanlan Geng ${ }^{1,8^{*}}$, \\ Wanfu $X u^{1,8^{*}}$ and Sitang Gong ${ }^{1,8^{*}}$
}

\begin{abstract}
Background: Mast cells (MCs) have been found to play a critical role during development of inflammatory bowel disease (IBD) that characterized by dysregulation of inflammation and impaired intestinal barrier function. However, the function of MCs in IBD remains to be fully elucidated.

Results: In our study, we used exosomes isolated from human mast cells-1 (HMCs-1) to culture with NCM460, HT-29 or $\mathrm{CaCO} 2$ of intestinal epithelial cells (IECs) to investigate the communication between MCs and IECs. We found that MCs-derived exosomes significantly increased intestinal epithelial permeability and destroyed intestinal barrier function, which is attributed to exosome-mediated functional miRNAs were transferred from HMCs-1 into IECs, leading to inhibit tight junction-related proteins expression, including tight junction proteins 1 (TJP1, ZO-1), Occludin (OCLN), Claudin 8 (CLDN8). Microarray and bioinformatic analysis have further revealed that a panel of miRNAs target different tight junction-related proteins. Interestingly, miR-223 is enriched in mast cell-derived exosome, which inhibit CLDN8 expression in IECS, while treatment with miR-223 inhibitor in HT-29 cells significantly reversed the inhibitory effect of HMCs-1-derived exosomes on CLDN 8 expression. Most importantly, enrichment of MCs accumulation in intestinal mucosa of patients with IBD compared with those healthy control.
\end{abstract}

Conclusions: These results indicated that enrichment of exosomal miR-223 from HMCs-1 inhibited CLDN8 expression, leading to destroy intestinal barrier function. These finding provided a novel insight of MCs as a new target for therapeutic treatment of IBD.

Keywords: Mast cells, Exosomes, miR-223, Claudin 8, Inflammatory bowel disease

\section{Background}

Inflammatory bowel disease (IBD) is a chronic gastroenterological inflammatory disease, and increasing

\footnotetext{
*Correspondence: genglan_2001@hotmail.com; xushi123@gmail.com; gongsitang@hotmail.com

${ }^{\dagger}$ Musheng Li, Junhong Zhao and Meiwan Cao contributed equally to this work

${ }^{1}$ Department of Gastroenterology, Guangzhou Women and Children's Medical Center, Guangzhou Medical University, Guangzhou 510623, China

Full list of author information is available at the end of the article
}

evidences have demonstrated that the mechanism of the pathogenesis in IBD is associated with dysfunction of intestinal epithelial barrier [1]. Imbalance between proinflammatory and anti-inflammatory effect in intestinal mucosa is major cause in development of IBD. Interestingly, the hallmark of IBD is a dysregulated intestinal immune response in which mast cells (MCs) accumulate in the inflamed gut of IBD patients [2]. Meanwhile, the report by Vivinus et al. [3] showed that increased MCs numbers were counted in the colon of both Crohn's Disease (CD) and Ulcerative colitis (UC) patients compared

(c) The Author(s) 2020. This article is licensed under a Creative Commons Attribution 4.0 International License, which permits use, sharing, adaptation, distribution and reproduction in any medium or format, as long as you give appropriate credit to the original author(s) and the source, provide a link to the Creative Commons licence, and indicate if changes were made. The images or other third party material in this article are included in the article's Creative Commons licence, unless indicated otherwise in a credit line to the material. If material is not included in the article's Creative Commons licence and your intended use is not permitted by statutory regulation or exceeds the permitted use, you will need to obtain permission directly from the copyright holder. To view a copy of this licence, visit http://creativeco mmons.org/licenses/by/4.0/. The Creative Commons Public Domain Dedication waiver (http://creativecommons.org/publicdomain/ zero/1.0/) applies to the data made available in this article, unless otherwise stated in a credit line to the data. 
to that in healthy controls, while activation of MCs are suggested by increased expression or release of MCs mediators in the mucosa, including proteases [4], histamine [5], chemokines and cytokines [6], leading to the attraction of inflammatory cells, changes in barrier function, tissue remodeling etc. However, the specific function of MCs in IBD development remains to be elucidated, especially the aberrant interactions between MCs and IECs or others underlying resident cells, which is fundamental for immunopathological regulation.

Intercellular communication is critical event to elicit efficient cell biology to aggravate or alleviate the process of IBD. In addition to various of inflammatory factors, small extracellular vesicles contain multifarious cargos such as proteins, mRNAs, InRNA and miRNAs, also known as exosomes, which could be released by MCs to suppress allergic reactions by binding to immunoglobulin $\mathrm{E}$ (IgE) [7] and active T cells via bioactive lipids like phospholipid scramblase, fatty acid binding protein phospholipases [8], and promote dendritic cell and B cells proliferation, maturation and cytokine secretion through CD40, CD80 and CD86 [9]. These studies suggested that MCs aggravated intestinal inflammation by triggering immunoreaction among immune cells to promote development of IBD. While the intestinal epithelial barrier is crucial for maintaining the intestinal homoeostasis because of its location between the luminal bacteria and the host's innate immune system. Tight junction-related proteins, including tight junction proteins 1 (TJP1, ZO-1), Occludin (OCLN), the junctional adhesion molecule (JAM) [10] and CLDN family [11], are the main components of the intestinal epithelial barrier, and they play an important role in controlling cellular polarity and adhesion [12]. Among these, CLDNs, in particular CLDN 8 , have been regarded as backbone of intestinal barrier and reported to be the most important related to IBD and diminished in IBD patients [13-15], which further focused us to analyze the potential relationship between MCs and tight junction proteins, especially CLDN 8, in IECs.

Evidences for the regulation of IBD by altered microRNA (miRNAs) is increasing [14, 16-18]. MiRNAs are a group of small, noncoding, endogenous RNAs that negatively regulate target genes, usually by imperfect complementation sequence pairing to the $3^{\prime}$ untranslated region (UTR) of the target genes, leading to mRNA cleavage and translational repression [19]. In this study, we revealed that MCs destroys intestinal barrier function via reduction of tight junction-related proteins, major in changes of CLDN 8 expression, mediated by exosomal miR-223, leading to increase epithelial permeability and contribute to development of IBD. Enrichment exosomal of miR223 in vesicles are secreted, transferred and internalized by IECs, resulting in suppressing CLDN 8 expression. These results demonstrate that, by targeting CLDN 8 in recipient cells, MCs-derived extracellular miR-223 is able to destroy intestinal barrier integrity to facilitate disease progression.

\section{Materials and methods \\ Cell cultures}

The IECs, including a normal human colon mucosal epithelial cell line NCM460, and human colorectal adenocarcinoma cell line HT-29 and $\mathrm{CaCO} 2$, were obtained from the ATCC and the human mast cells (HMCs-1) was purchased form Jiniou Company. HMCs-1 were maintained in Dulbecco's Modified Eagle Medium (DMEM) supplemented with $10 \%$ exosome depleted fetal bovine serum (FBS), $100 \mathrm{units} / \mathrm{ml}$ penicillin, $100 \mu \mathrm{g} / \mathrm{ml}$ streptomycin, $2 \mathrm{mM} \mathrm{L}$-glutamine and $1.2 \mathrm{mM}$ alpha-thioglycerol (all reagents were from Sigma-Aldrich, St Louis, MO, USA). NCM460, HT-29 and CaCO2 cells were routinely maintained in DMEM medium (HyClone Laboratories, Inc.) supplemented with $10 \% \mathrm{FBS}, 100$ units/ml penicillin and $100 \mu \mathrm{g} / \mathrm{ml}$ streptomycin. All cells were cultured at 37 ${ }^{\circ} \mathrm{C}$ in a humidified atmosphere of $5 \% \mathrm{CO} 2$.

\section{Antibodies}

The antibodies including c-kit (Catalog:A0357); ZO-1 (Catalog:A0659); Occludin (Catalog:A2601); Claudin1 (Catalog:A2196); GAPDH (Catalog:AC033); CD63 (Catalog:A5271); TSG101 (Catalog:A1692); Actin (Catalog:AC026); Calnexin (Catalog: A4846) were purchased from Abclonal Company. GOLGA2/GM130 (Catalog:11308-1-AP) was purchased from Proteintech Company. Histone 3 (Catalog: D2B12) was purchased from Cell Signaling Technology Company.

\section{Isolation of exosomes}

Exosomes were isolated from the supernatant of HMCs-1 as described in Xiao et al. [20] study, briefly, cell supernatant were harvested, centrifuged at $300 \times g$ for $10 \mathrm{~min}$ to eliminate cells and at $16,500 \times g$ for $20 \mathrm{~min}$, followed by filtration through $0.2 \mu \mathrm{m}$ filter (Sarstedt, Numbrecht, Germany) to remove cellular debris and larger vesicles. Exosomes were pelleted by ultracentrifugation at $120,000 \times g$ for $70 \mathrm{~min}$. Exosomes were measured for their protein content using the BCA protein assay kit (Thermo Scientific Pierce, Rockford, IL, USA).

\section{PKH67-labelled exosome of HMCs-1 uptake into IECs}

After isolation from HMCs-1 culture medium, exosomes were labelled with PKH67 fluorescent cell linker (SigmaAldrich, St. Louis, MO) according to manufacturer's instructions. $10 \mu \mathrm{g}$ of the PKH67-stained exosomal solution or control solution were added into DMEM to 
co-culture with IECs in slides for $24 \mathrm{~h}$ respectively. After washing and fixation with $4 \%$ formaldehyde solution for 15 min, the slides were washed with PBS, stained nuclei using a Prolong Gold Antifade Reagent with 4',6-diamidino-2-phenylindole (DAPI; Life Technologies), covered with coverslips and visualized under a confocal microscope (LSM710; Carl Zeiss, Oberkochen, Germany).

\section{Generation of stable HMCs-1 Cell line}

The lentivirus vectors Lv-miRNA-223 mimics, LvmiRNA-NC were purchased from Genepharma. Puromycin was purchased from Sigma and used to select for stably cell lines.

\section{Immunofluorescence microscopy}

Immunofluorescence (IF) was performed in our previous studies $[1,19]$. Cells plated on coverslips were pretreated with $0.2 \%$ Triton X-100 in PBS (PBST) for 5 min after fixation with $4 \%$ paraformaldehyde followed by treatment with glycine for another 5 min to stop fixation with, the slides were blocked with $1 \%$ bovine serum albumin in PBST for $30 \mathrm{~min}$ followed by incubation with primary antibodies for overnight at $4{ }^{\circ} \mathrm{C}$, and then washed with PBST for 3 times and incubated with secondary antibodies for another $1 \mathrm{~h}$ at room temperature. DNA was stained with 4,6-diamidino2-phenylindole (DAPI) for 2-3 min and covered with glass. Images were captured and analyzed under a confocal laser scanning microscope.

\section{Transfer of miRNA to IECs}

To determine if the miRNAs could be transferred via exosomes, exosomes $(10 \mu \mathrm{g})$ were added to IECs to incubate for $24 \mathrm{~h}$. The total RNA was extracted and miRNA was detected using miRNA Kit from Shanghai Genepharm, and real-time polymerase chain reaction (PCR) was performed to detect.

\section{Western blot analysis}

Western blotting was performed in our previously studies [19], the total proteins were collected and subjected to SDS-PAGE, transferred to nitrocellulose membrane (Bio-Rad Laboratories). After blocked with $5 \%$ bovine serum albumin (BSA) in PBS with tween 20 (PBST) for 1 $h$, the membrane was then incubated with indicated primary antibody for overnight at $4{ }^{\circ} \mathrm{C}$ followed by incubation with a horseradish peroxidase secondary antibody (Jackson ImmunoResearch) for $1 \mathrm{~h}$ at room temperature. Proteins were detected using an enhanced chemiluminescence (Perkin Elmer).

\section{Real-time PCR}

As described in our previously study, total RNA was extracted using Trizol (life technologies), while miRNAs expression was detected using a SYBR Green I Real-Time PCR kit (GenePharma) by an Applied Biosystems StepOnePlus system.

\section{Clinical sample}

The intestinal tissue was drawn from each patient by electronic colonoscopy after we got the informed consent from the patients diagnosed with IBD. This study was conducted in a cohort of child patients with IBD in Guangzhou Women and Children's Medical Center approved by the Medical Ethical Review Board, named Scientific Research Committee of Guangzhou Women and Children's Medical Center.

\section{Immunohistochemistry}

Immunohistochemistry (IHC) were performed as described in our previous work. The sections were deparaffinized, rehydrated, blocked with goat serum (ThermoFisher, Catalog: 16210064) and incubated with indicated antibody at $4{ }^{\circ} \mathrm{C}$ overnight, the bound antibodies were then visualized using diaminobenzidine as a chromogen, and the slides were counterstained with hematoxylin. The area of positive staining was measured in six different images taken at $400 \times$ magnification on each slide and quantified using Image-Pro Plus 6.0 software (Media Cybernetics).

\section{Statistical analysis}

All analysis was conducted using GraphPad Prism V software. A P value $<0.05$ was considered statistically significant. Statistical differences among groups were determined by Student's t- test, one sample t-tests were used to determine the significance of between-group differences in RT-qPCR results.

\section{Results}

HMCs-1-secreted exosome destroy IECs barrier function

To explore the novel way of MCs in development of IBD, HMCs- 1 and $\mathrm{CaCO} 2$ and NCM460 of IECs were employed as models for studying HMCs-1-secreted exosomes and miRNAs. As described in Zhou et al. [21] study, purified exosomes, a size range of 30 to $100 \mathrm{~nm}$ and morphology confirmed by transmission electron microscope (TEM) (Additional file 1: Fig. S1A), isolated from HMCs-1 conditioned media displayed exosome marker CD63 and TSG101, but not GM130, Histone 3 and Calnexin(Additional file 1: Fig. S1B), by western blotting analysis to confirm that these vesicles were exosomes (Fig. 1a), implying exosomes were efficiency extracted. Next, we focused our attention on IECs in this study for 


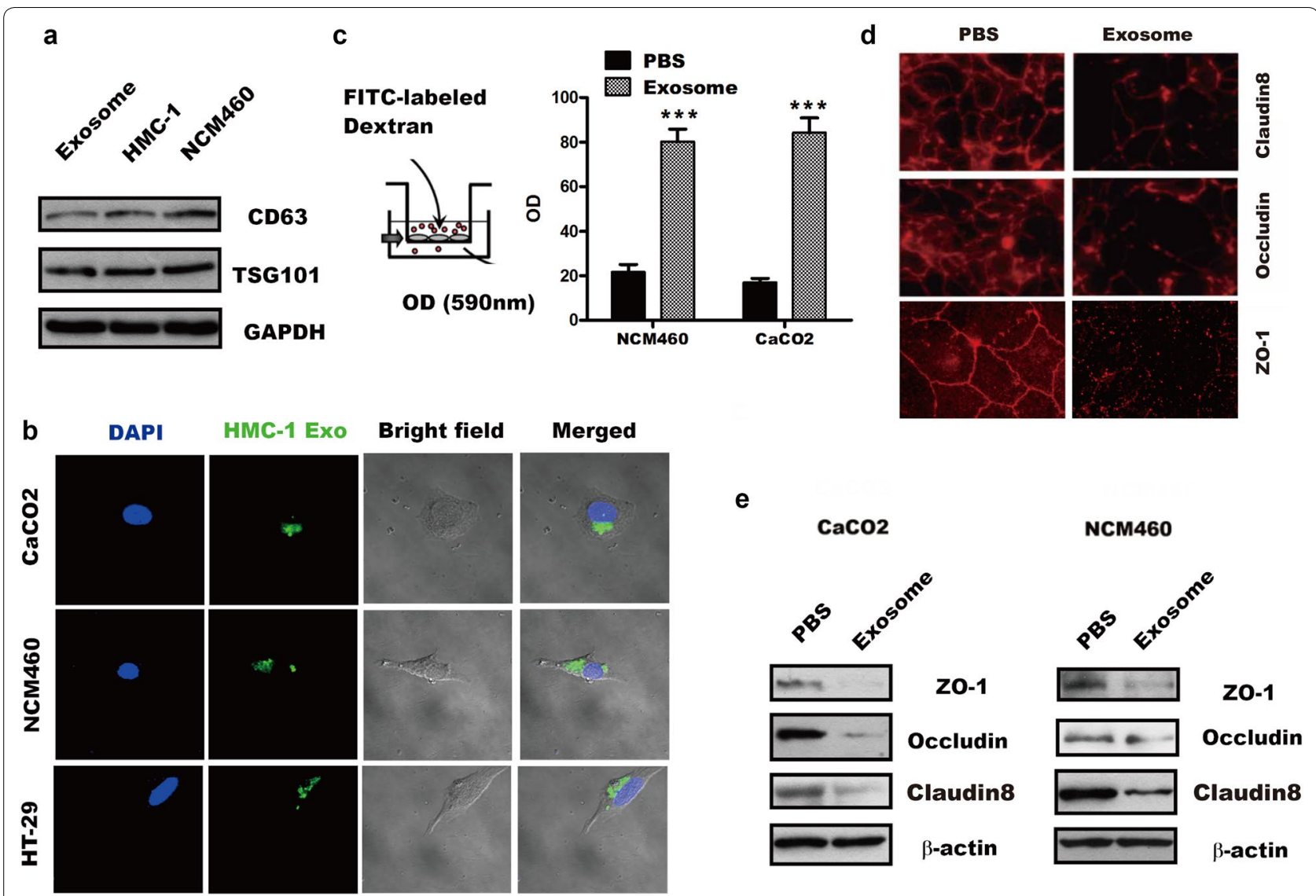

Fig. 1 MCs-secreted exosomes were internalized and destroyed intestinal barrier function. a Western blotting analysis of indicated proteins in HMCs-1, NCM460 and exosomes. b Indicated IECs were incubated with PKH67-labelled exosomes (green) for $24 \mathrm{~h}$ before fluorescent and phase contrast images were captured. c The permeability of treated $\mathrm{CaCO} 2$ monolayers grown on $0.4 \mathrm{~mm}$ filters was measured by the appearance of rhodamine-dextran, which was added to the top well at the beginning of the experiment, in the bottom well during a $1 \mathrm{~h}$ time course. The absorbance at the $1 \mathrm{~h}$ time point was compared with the PBS (control) condition. ${ }^{* *} \mathrm{p}<0.001$. d IF analysis of CLDN 8, OCLN and ZO-1 in CaCO2 cells treated with or without exosomes. e Western blotting analysis of the changes of tight junction protein in $\mathrm{CaCO} 2$ and NCM460 cells treated as indicated

their critical barrier function in response to HMCs-1-derived exosome. Interestingly, when exosomes labelled with the fluorescent dye PKH67 were incubated with IECs, the recipient cells exhibited high up-take efficiency as indicated by fluorescence microscopy (Fig. 1b), and in vitro permeability assay were performed by measuring the traversing of rhodamine-labeled dextran probes through $\mathrm{CaCO} 2$ monolayers growing on 0.4- $\mathrm{mm}$ filters. The results showed that treatment of the intestinal barrier with HMCs-1 exosomes induced passage of the fluorescent probes from the top to the bottom, with about 4 times increment of the OD value compared to PBS group (Fig. 1c). These findings suggested HMCs-1-derived exosomes were internalized and increased intestinal permeability.

To further identify the potential changes of tight junction-related proteins, including TJP1, OCLN, and CLDNs, in response to HMCs-1-secreted exosome treatment. We co-cultured $\mathrm{CaCO} 2$ or NCM460 cells in DMEM medium, with $10 \%$ exosome-depleted FBS, with $20 \mu \mathrm{g} / \mathrm{ml}$ exosomes for $72 \mathrm{~h}$. Control cells (PBS group) were $\mathrm{CaCO} 2$ or NCM460 cells that were not exposed to exosomes. As shown in Fig. 1d, $\mathrm{CaCO} 2$ monolayers treated with exosomes drastically reduced ZO-1, OCLN and CLDN 8 by immunofluorescence analysis, the western blotting (Fig. 1e) and quantitation (Additional file 1: Fig. S1C) results also showed ZO-1, OCLN and CLDN8 were significantly reduced in $\mathrm{CaCO} 2$ and NCM460 treated with exosome compared with that PBS group. Taken together, these results indicated the HMCs-1 exosome destroyed intestinal barrier function by inhibition of ZO-1, OCLN and CLDN 8. 


\section{MiR-223 is carried by exosomes and suppresses CLDN 8 in IECs}

It is well known that exosomes, carrying miRNAs and other substances, could be internalized and regulated biological function of recipient cells. Interestingly, in line with Chevillet et al. [22] study, a panel of miRNAs, especially enrichment of miR-223, was detected in HMCs-1 exosomes listed in Table 1 by miRNA microarray (Additional file 2: Table S1). Upon these findings, we tried to seek whether miR-223 were delivered from MCs to IECs via exosome to induce IBD. The exosome isolated from HMCs-1 infected with miR-223 lentivirus were co-cultured with IECs for analysis the level of miRNAs expression. As shown in Fig. 2a, after 24 h co-culture, exosome containing miR-223 (green) were located in cytosolic of HT-29 and NCM460 of IECs under fluorescence capture (left panel). The analysis of exosome-treated HT-29 and NCM460 cells by (Flow Cytometry) FCM showed that HT-29 and NCM460 cells internalized exosomal miR223, respectively (right panel).

Next, the total RNA was extracted to detect indicated miRNA expression, the result revealed that the level of most miRNAs, especial miR-223, in HT-29 cells with exosomes treatment were significantly higher than that without exosomes co-culture, which indicated that
MCs-derived exosome. To further confirm exosomemediated miRNA destroy tight junction-related proteins via these regulatory miRNAs, an antagonized experiment for top three miRNAs was performed to confirm the effect of exosomes could be abolished by transfecting the recipient cells with miRNA inhibitor. The results showed that inhibition of miR-223 in HT-29 cells by transfection with miR-223 inhibitor significantly reversed the inhibition of exosome on CLDN 8 expression, leading to decrease intestinal epithelial permeability (Fig. 2c), the similar results were obtained in IECs treatment with miR-21 and miR-16 (Additional file 1: Fig. S1D). Taken together, we concluded that miRNAs can be delivered by the exosomes of HMCs-1 and suppress the expression of tight junction-related proteins.

\section{The relationship between tight junction proteins and exosomal miRNAs}

Dysregulated Inflammation-triggered miRNAs could promote cancer cell proliferation through PI3K/Akt. Such as miR-223 is upregulated in human colorectal cancer(CRC), IBD, and the IL-10 knockout mouse model of IBD [23], while miR-34a, miR-142-5p, miR-146a, miR148a, and miR-223 were altered in AOM/DSS-regulated miRNAs and human IBD, and upregulated in three DSS

Table 1 Schematic representation of tight junction-related protein affected by miRNAs

\begin{tabular}{llll}
\hline miRNA & Targets & miRNA & Targets \\
\hline hsa-miR-223 & CLDN8 [14] & hsa-miR-212 & CLDN, JAMC, TJP1 [41, 42] \\
hsa-miR-21 & OCLN, CLDN1, CLDN5 [43-45, 39] & hsa-miR-29a & CLDN1 [46] \\
hsa-miR-16 & ZO-1, OCLN, CLDN2 [47, 48] & hsa-miR-18a & ZO-1, OCLN, CLDN5 [49, 50] \\
hsa-miR-23a & ZO-1 [40] & hsa-miR-146a & CLDN1, OCLN, JAMA [51] \\
hsa-miR-320a & JAMA [52] & hsa-miR-34c-5p & ZO-1, OCLN [53] \\
hsa-miR-191 & ZO-1 [54] & hsa-miR-34c-3p & ZO-1, OCLN [53] \\
hsa-miR-99b & CLDN11 [55] & hsa-miR-181a & ZO-1, OCLN, CLDN5 [28] \\
hsa-let-7b & OCLN, ZO-1 [56] & hsa-miR-122 & ZO-1, ZO-3, OCLN [57, 58] \\
hsa-miR-132 & CLDN1, JAM3, TJP1 [41,59] & hsa-miR-143 & ZO-1, ZO-3, OCLN, CLDN5 [60, 61] \\
hsa-miR-101 & VE-Cadherin [62] & hsa-miR-21-3p & OCLN, CLDN5, ZO-1 [63] \\
hsa-miR-15a & ZO-1, OCLN [48] & hsa-miR-125b & CLDN2 [47] \\
hsa-miR-210 & OCLN [64] & hsa-miR-200b & OCLN, CLDN5, CLDN1, ZO-1 [65, 66] \\
hsa-miR-34a & ZO-1, OCLN, CLDN5 [67] & hsa-miR-96 & ZO-1 [68] \\
hsa-miR-150 & CLDN5 [69] & hsa-miR-429 & OCLN [70] \\
hsa-miR-107 & ZO-1, OCLN, CLDN5 [71] & hsa-miR-30a & CLDN1, CLDN2, CLDN3 [72] \\
hsa-miR-144 & OCLN, ZO-1, CLDN5 [73] & & \\
\hline
\end{tabular}

The miRNAs detected in MCs-derived exosomes have been reported to target different tight junction proteins. The enrichment of miRNA CT value was listed in Additional file 1: Table S1

exosomes are potential mediator of miRNAs in communication between MCs and HT-29 cells (Fig. 2b). Based on these findings, we could conclude that a serial of miRNAs, including miR-223, were transferred into IECs via cycles confirmed by PCR experiments [23, 24]. As shown in Table 1 , in addition to the report by Wang et al. [14] showed that CLDN8 is a target of miR-223, respectively, while ZO-1 is showed a target of miR-101 [25] and 


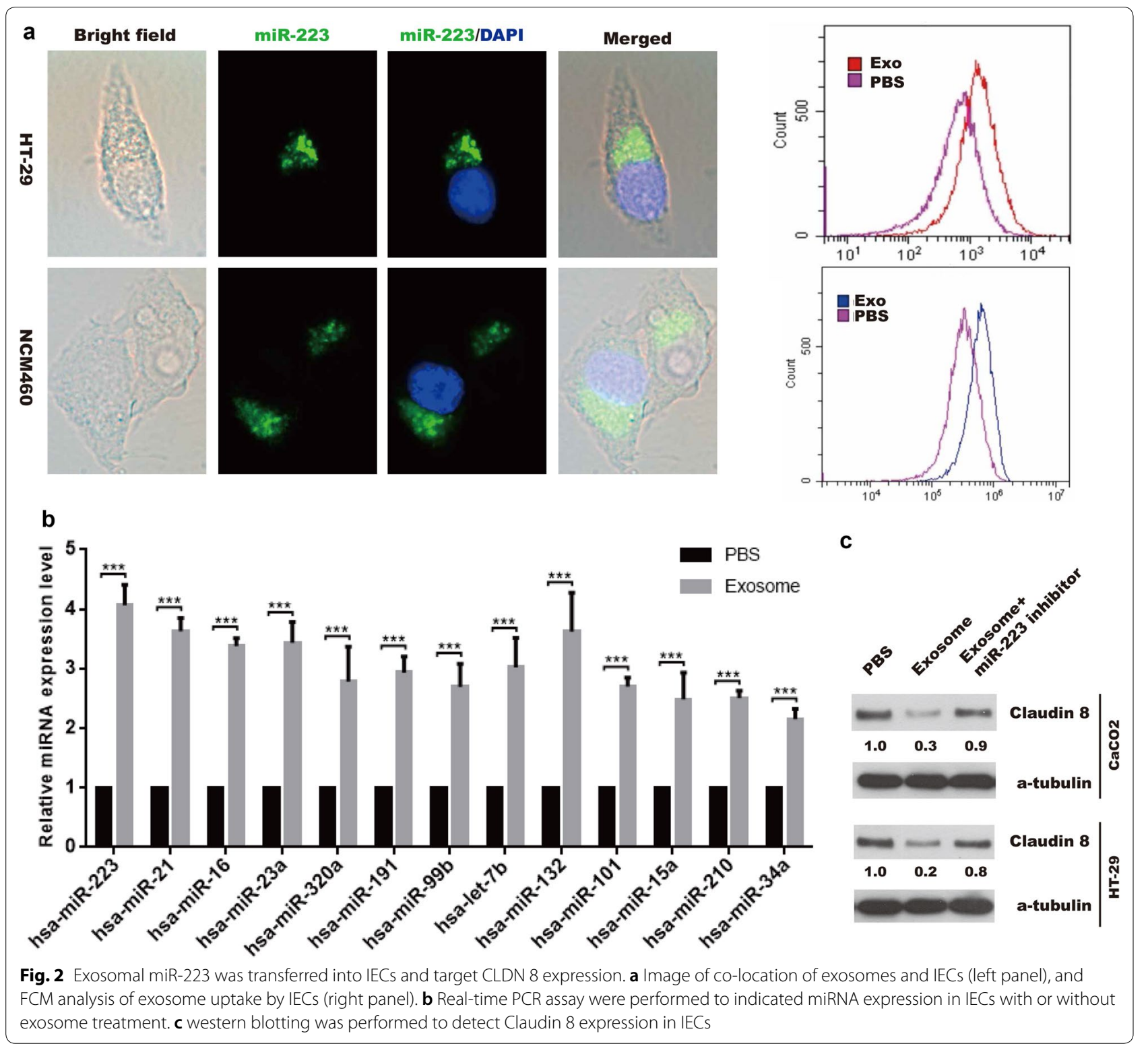

miR-105 [26], the studies also found that OCLN, CLDN5 and ZO-1 are targets of miR-34a [27] and miR-181a [28].

Based on the outcomes described above, we inferred that the tight junction-related proteins, including ZO-1, OCLN, and CLDN family, were regulated by a several of miRNA analyzed by bioinformatics and verified by multiple studies, implying miRNAs play a role as pro-IBD in IBD. What's more, the studies also showed that miR-223 is significantly increased in intestinal mucosa tissues in patients with IBD compared with normal tissues after measuring the level of miR-223 by fluorescence in situ hybridization (FISH) [14], while miR-21 and other miRNAs were also reported to target different genes involved in intestinal barrier function in other studies listed in Table 1. These finding indicated that exosomal miRNAs has a critical role in regulating intestinal barrier function in development of IBD.

\section{Enrichment of MCs numbers in patients with IBD correlates} with the severity of intestinal inflammation

The above results have suggested that MCs exosomal miRNAs target tight junction-related proteins in IECs, leading to increase intestinal permeability and destroy intestinal barrier function, which focus us to explore the relationship between MCs and intestinal barrier function. As shown in Fig. 3a, enrichment of MCs stained 
with C-kit by IHC in intestinal mucosa of patients with IBD compared with healthy control, while miR-223, an abundant of MCs exosomal miRNA, is also increased in active UC and CD by real-time PCR assay (Fig. 3b). These findings implied enrichment of MCs is another source of miR-223, leading to target CLDN8 expression.

\section{Discussion}

Despite multiple studies have showed MCs play an active role in the pathogenesis of a variety of pediatric gastrointestinal (GI) disorders, including epithelial function (water and electrolyte secretion, tight junction/epithelial barrier integrity), endothelial function (blood flow, vessel contraction, endothelial permeability, coagulation/ fibrinolysis), cell influx into tissue (neutrophils, eosinophils, lymphocytes), neuroenteric function (intestinal peristalsis, pain mediation), and tissue transformation (wound healing, fibrosis) [29], the exact mechanism by which MCs mediators participate in IBD remains to be fully elucidated. In this study, as shown in Fig. 4, our results further demonstrated that HMCs-1 triggered the development of IBD via exosomal miRNA, including miRNA-223, miR-105 and so on. MCs-derived exosomes inhibited tight junction-related proteins, leading to increase intestinal epithelial permeability and destroy barrier function by western blotting, IF and in vitro permeability assay. These findings suggested a novel pathway of MCs contributed to development of IBD.

Accumulating evidences indicated that the function of MCs in IBD is more complicated than originally thought, Animal and human clinical studies suggested that the contribution of these cells includes regulating epithelium permeability, immune signal transmittance, maintenance and resolution of inflammatory responses, and subsequent tissue remodeling [2, 30-34]. What's more, the study showed that the increase of MCs infiltration near the epithelium resulted in a favorable microenvironment that expressed more beneficial proteins including ZO-1, FGF2, ANGPTL2, REG3 $\gamma$, and REG3 $\beta$, which are involved in signal transduction, cell growth, tissue repair, and homeostasis maintenance [2]. In addition to Wilcz-Villega et al. [35] study showed that a MCs tryptase increased permeability to macromolecules and decreased resistance in a $\mathrm{CaCO} 2$ epithelial cell layer system, our results further demonstrated that MCs-derived exosomes reduced ZO-1, OCLN and CLDN1 expression, leading to destroy intestinal epithelial barrier function, which provided a novel pathway of MCs in IBD. In intestinal inflammatory microenvironment, the interactions between MCs and IECs may result in aggravating intestinal inflammation and poor patient prognosis. Preventing intestinal epithelium-MCs communication may alleviate IBD progression, which provided an important treatment target for IBD therapy. Active MCs contributed to aggravate inflammation reaction and induced colitis-related cancer by releasing the classical pro-angiogenic and proinflammatory factors including VEGF, FGF-2, PDGF, and IL-6, and nonclassical pro-angiogenic factors proteases including tryptase and chymase. In addition to secreted soluble mediators, exosomes, a membrane vesicles contains numerous proteins, lipids, and even nucleic acids, play an important role in intercellular communication, which could induce transient or persistent phenotypic changes in the recipient cell $[36,37]$ and the role of exosomal miRNA in inflammation and barrier function has been highlighted [38].

Interestingly, in line with our results, the Chevillet et al. [22] study have also revealed that different enrichment of MCs exosomal miRNA listed in Table 1 by quantitative and stoichiometric analysis, for example,

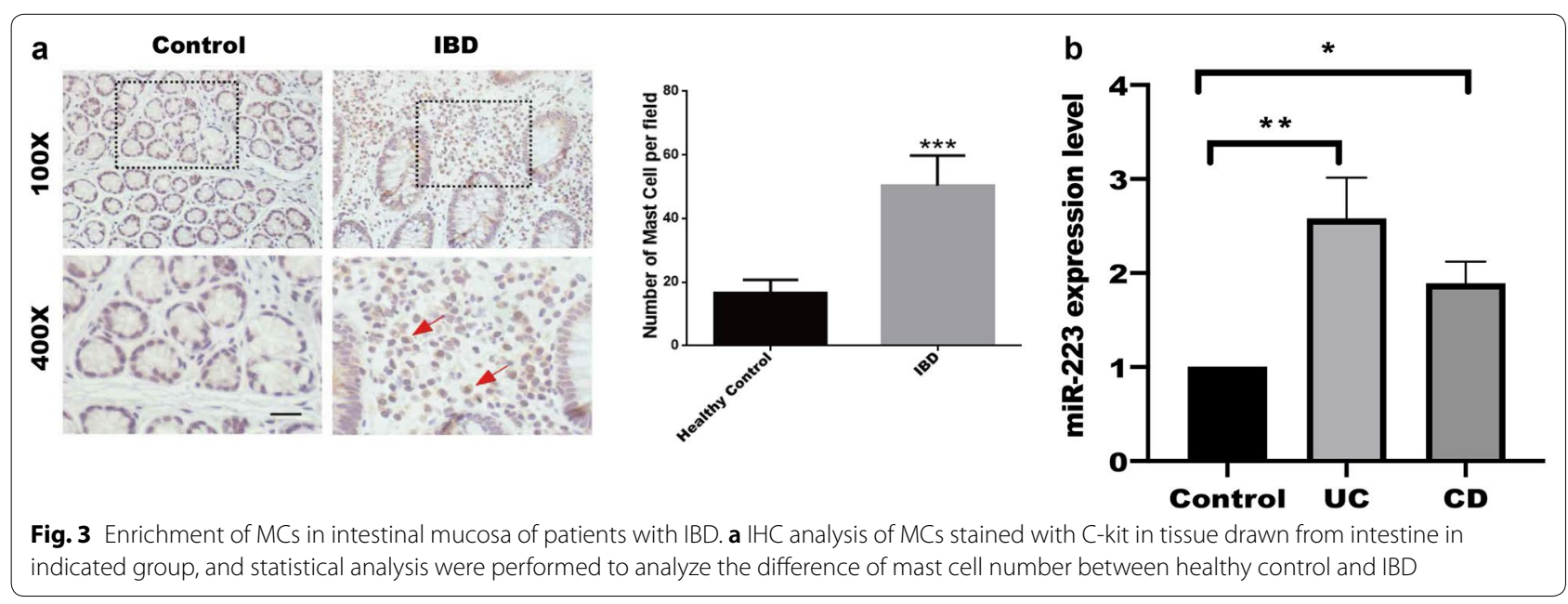




\section{Mast Cells}

\section{IECS}
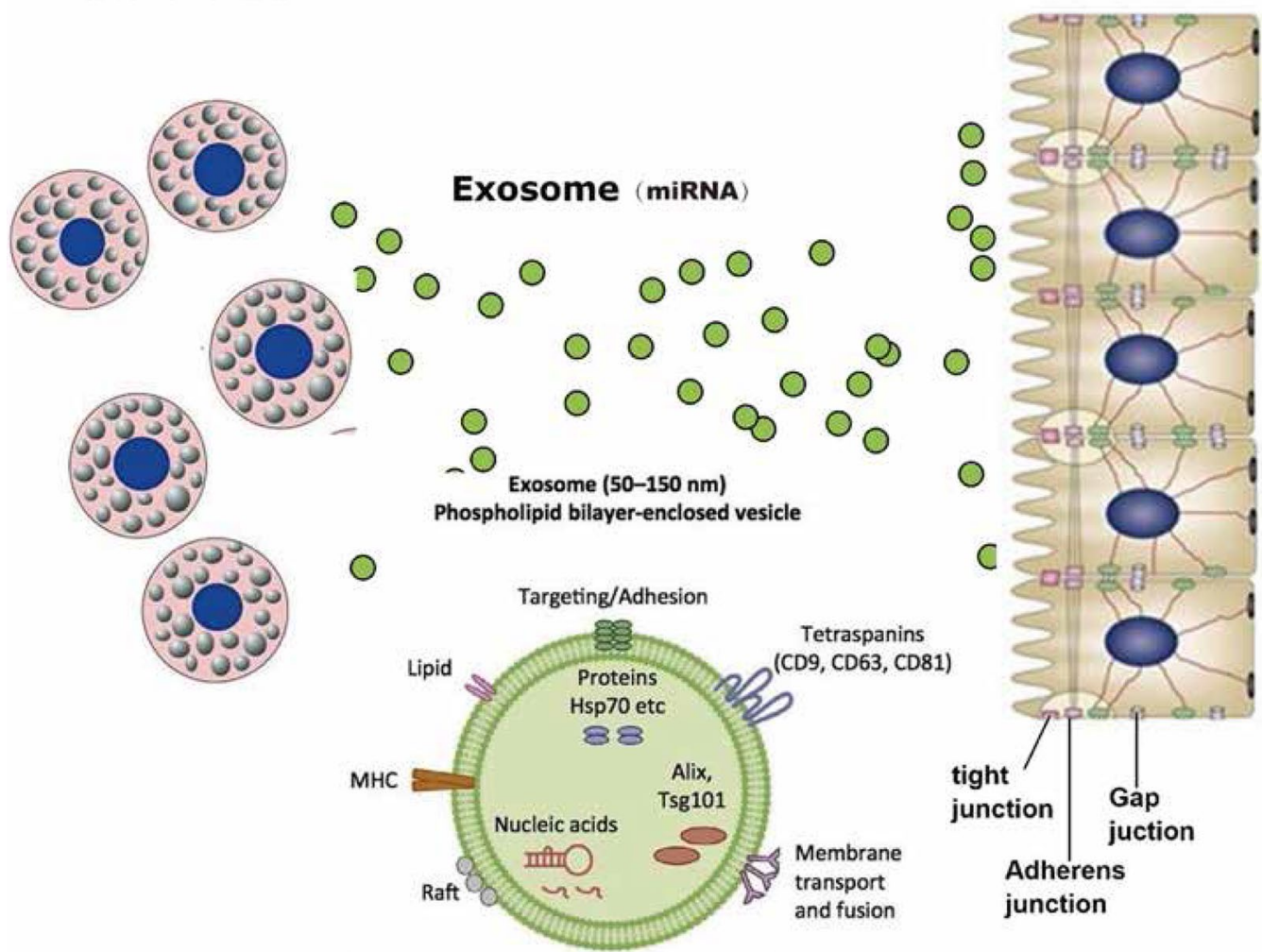

Fig. 4 Schematic illustration for MCs-derived miRNA destroys by inhibition of tight junction-related proteins in IECs. Enrichment of miRNA in MCs were transferred into IECs via exosomes, and internalized exosomal miRNAs suppressed tight junction-related proteins expression, including ZO-1, Occludin and Claudin, leading to increase intestinal epithelial permeability and destroy intestinal barrier function

miR-223, a pro-inflammatory miRNA, has been verified to target CLDN8 in IL-23 pathway [14], while inversely correlation between miR-21 expression and the levels of intestinal tight junction proteins OCLN and CLDN1 in intestinal ischemia-reperfusion group, compared with sham group [39], and lung cancer cells exosomal miR-23a inhibited tight junction protein ZO-1, thereby increasing vascular permeability and cancer transendothelial migration [40]. All these findings suggested that MCs exosomes have a global influence on intestinal barrier function by regulation of tight junction-related proteins. However, further work is required to elucidate the changes of inflammatory factors in IECs. Taken together, these findings provided an understanding that miRNAs are increased in patients with IBD. Thus, therapies targeting $\mathrm{MCs}$, and in combination with existing conventional therapies, may serve as an effective treatment for active stage patients with IBD.

\section{Conclusions}

In conclusion, this study demonstrated that functional exosomal miRNAs can be transported from HMCs-1 to IECs. MCs-derived exosomal miRNAs shuttled into IECs, and destroyed intestinal epithelial barrier function by targeting to inhibit tight junction-related proteins. Thus, our study provided novel insights into the mechanisms of cell-cell interactions through which MCs regulated the intestinal epithelial barrier function via the exosomal miRNAs.

\section{Supplementary information}

Supplementary information accompanies this paper at https://doi. org/10.1186/s40659-020-00279-2.

Additional file 1: Figure S1. Inhibition of miR-21/16 reversed the effect of exosome on IECS.

Additional file 2: Table S1. Enrichment of miRNAs derived by human mast cells was listed by miRNA array. 


\section{Abbreviations}

CLDN: Claudin; CD: Crohn's disease; CRC: Colorectal cancer; DAPI: 4,6-diamidino-2-phenylindole; DMEM: Dulbecco's Modified Eagle Medium; FCM: Flow cytometry; FISH: Fluorescence in situ hybridization; GI: Gastrointestinal; HMC-1: Human mast cells-1; IBD: Inflammatory bowel disease; IECs: Intestinal epithelial cells; IgE: Immunoglobulin E; JAM: Junctional adhesion molecule; MCs: Mast Cells; microRNA: MiRNA; OCLN: Occludin; PCR: Polymerase chain reaction; TEM: transmission electron microscope; UC: Ulcerative colitis; UTR: untranslated region; ZO-1: Tight junction protein 1.

\section{Acknowledgements}

We would like to thank doctors in department of gastroenterology for kindly supporting. We also thank Dr. Yuanwen Xie, Dr. Songyu Li and Dr. Kejian Zou for supporting in data analysis and funding.

\section{Authors' contributions}

STG, WFX and LLG conceived and designed the experiments, YC, JHZ, MSL, MWC, RTL, GHC, SYL, YWX, LH, KJZ, JX, YXX and MMS performed experiments and analyzed data, WFX, LLG and STG wrote the manuscript, and all authors read and approved the final manuscript.

\section{Funding}

This research was funded by National Natural Science Foundation of China (No.81770552, No.81860101), Natural Science Foundation of Guangdong Province (No.2017A030313838, No.2018A0303130175), Medical Science and Technology Foundation of Guangdong (No.A2018395), Guangzhou Municipal Science and Technology Project (No.201804010148, No.201904010485), Guangzhou Municipal Health and Family Planning Commission (No.20191A010024), Funding of Guangzhou Institute of Pediatrics/Guangzhou Women and Children's Medical Center (No.IP-2016005, No.IP-2018-009, Pre-NSFC-2019-005), Funding of cooperation projects between Guangzhou Women and Children's Medical Center and Sun Yat-Sen University(No.201704020223), Natural Science Foundation of Hainan Province (No.2819QN372). Clinical key specialty and construction of cultivate the key subject of Guangzhou Women and Children's Medical Center (No.170000105), High-level talents of Hainan natural science foundation (No.2019RC366).

\section{Availability of data and materials}

The datasets generated during and/or analyses during the current study are available from the corresponding author on reasonable request.

\section{Ethics approval and consent to participate}

Human study: Based on the declaration of Helsinki as reflected in a prior approval by the institution's human research committee, this study was conducted in a cohort of 77 patients with IBD and 19 healthy control in Guangzhou Women and Children's Medical Center from 2016 to 2018 approved by Guangzhou Women and Children's Medical Center animal care and use committee. Written informed consent was given by the caregiver of the child for his clinical records used, which are not publicly available since the database is currently not anonymous and contains all patient's name; however, it could be available upon request.

\section{Consent for publication}

Not applicable.

\section{Competing interests}

The authors declared that they have no competing interests.

\section{Author details}

1 Department of Gastroenterology, Guangzhou Women and Children's Medical Center, Guangzhou Medical University, Guangzhou 510623, China. ${ }^{2}$ Department of Clinical Laboratory, Qionghai Hospital of Traditional Chinese Medicine, Qionghai 571400, China. ${ }^{3}$ Department of Anorectal, Qionghai Hospital of Traditional Chinese Medicine, Qionghai 571400, China. ${ }^{4}$ Department of Cancer Biology and Therapeutics, School of Pharmacy and Pharmaceutical Sciences, Cardiff University, Wales CF103AT, UK. ${ }^{5}$ Department of Preventive Medicine, School of School of Public Health, Fujian Medical University, Fuzhou 350122, China. ${ }^{6}$ School of Marine Life Sciences, Ocean University of China, Qingdao, Shandong 266003, China. ${ }^{7}$ Department of General Surgery, Hainan General Hospital, Haikou, China. ${ }^{8}$ Guangzhou Institute of Pediatrics, Guangzhou
Women and Children's Medical Center, Guangzhou Medical University, Guangzhou 510623, China.

Received: 8 November 2019 Accepted: 9 March 2020

Published online: 24 March 2020

\section{References}

1. Zhang S, Xu W, Wang H, Cao M, Li M, Zhao J et al (2019) Inhibition of CREB-mediated ZO-1 and activation of NF-kappaB-induced IL-6 by colonic epithelial MCT4 destroys intestinal barrier function. Cell Prolif. 52(6):e12673.

2. He Z, Song J, Hua J, Yang M, Ma Y, Yu T, et al. Mast cells are essential intermediaries in regulating IL-33/ST2 signaling for an immune network favorable to mucosal healing in experimentally inflamed colons. Cell Death Dis. 2018;9(12):1173.

3. Nebot-Vivinus M, Harkat C, Bzioueche H, Cartier C, Plichon-Dainese R, Moussa L, et al. Multispecies probiotic protects gut barrier function in experimental models. World J Gastroenterol. 2014;20(22):6832-43.

4. Saadalla AM, Osman A, Gurish MF, Dennis KL, Blatner NR, Pezeshki A, et al. Mast cells promote small bowel cancer in a tumor stagespecific and cytokine-dependent manner. Proc Natl Acad Sci USA. 2018;115(7):1588-92.

5. Potts RA, Tiffany CM, Pakpour N, Lokken KL, Tiffany CR, Cheung K, et al. Mast cells and histamine alter intestinal permeability during malaria parasite infection. Immunobiology. 2016;221(3):468-74.

6. Eissmann MF, Dijkstra C, Jarnicki A, Phesse T, Brunnberg J, Poh AR, et al. IL-33-mediated mast cell activation promotes gastric cancer through macrophage mobilization. Nat Commun. 2019;10(1):2735.

7. Xie L, Liu T, Yao H, Wu L, Qiu W, Chen B, et al. Autoimmune inflammatory meningoencephalitis in a patient negative for glial fibrillary acidic protein-specific immunoglobulin G. Mult Scler Relat Disord. 2019;28:167-71.

8. Cheung KL, Jarrett R, Subramaniam S, Salimi M, Gutowska-Owsiak D, Chen YL, et al. Psoriatic T cells recognize neolipid antigens generated by mast cell phospholipase delivered by exosomes and presented by CD1a. J Exp Med. 2016;213(11):2399-412.

9. Skokos D, Botros HG, Demeure C, Morin J, Peronet R, Birkenmeier G, et al. Mast cell-derived exosomes induce phenotypic and functional maturation of dendritic cells and elicit specific immune responses in vivo. J Immunol. 2003;170(6):3037-45.

10. Laukoetter MG, Nava P, Lee WY, Severson EA, Capaldo CT, Babbin BA, et al. JAM-A regulates permeability and inflammation in the intestine in vivo. J Exp Med. 2007;204(13):3067-76.

11. Gunzel D, Yu AS. Claudins and the modulation of tight junction permeability. Physiol Rev. 2013;93(2):525-69.

12. Takaki Y, Hirai S, Manabe N, Izumi Y, Hirose T, Nakaya M, et al. Dynamic changes in protein components of the tight junction during liver regeneration. Cell Tissue Res. 2001;305(3):399-409.

13. Zeissig S, Burgel N, Gunzel D, Richter J, Mankertz J, Wahnschaffe U, et al. Changes in expression and distribution of claudin 2, 5 and 8 lead to discontinuous tight junctions and barrier dysfunction in active Crohn's disease. Gut. 2007;56(1):61-72.

14. Wang H, Chao K, Ng SC, Bai AH, Yu Q, Yu J, Li M, Cui Y, Chen M, Hu JF, Zhang S. Pro-inflammatory miR-223 mediates the cross-talk between the IL23 pathway and the intestinal barrier in inflammatory bowel disease. Genome Biol. 2016;17:58.

15. Clark PM, Dawany N, Dampier W, Byers SW, Pestell RG, Tozeren A. Bioinformatics analysis reveals transcriptome and microRNA signatures and drug repositioning targets for IBD and other autoimmune diseases. Inflamm Bowel Dis. 2012;18(12):2315-33.

16. Wu CP, Bi YJ, Liu DM, Wang LY. Hsa-miR-375 promotes the progression of inflammatory bowel disease by upregulating TLR4. Eur Rev Med Pharmacol Sci. 2019;23(17):7543-9.

17. Pei XF, Cao LL, Huang F, Qiao X, Yu J, Ye H, et al. Role of miR-22 in intestinal mucosa tissues and peripheral blood CD4+T cells of inflammatory bowel disease. Pathol Res Pract. 2018;214(8):1095-104.

18. Li M, Zhang S, Qiu Y, He Y, Chen B, Mao R, et al. Upregulation of miR-665 promotes apoptosis and colitis in inflammatory bowel disease by repressing the endoplasmic reticulum stress components XBP1 and ORMDL3. Cell Death Dis. 2017;8(3):e2699. 
19. Xu W, Zhang Z, Zou K, Cheng Y, Yang M, Chen H, et al. MiR-1 suppresses tumor cell proliferation in colorectal cancer by inhibition of Smad3mediated tumor glycolysis. Cell Death Dis. 2017;8(5):e2761.

20. Xiao H, Lasser C, Shelke GV, Wang J, Radinger M, Lunavat TR, et al. Mast cell exosomes promote lung adenocarcinoma cell proliferation-role of KIT-stem cell factor signaling. Cell Commun Signal. 2014;12:64.

21. Zhou M, Chen J, Zhou L, Chen W, Ding G, Cao L. Pancreatic cancer derived exosomes regulate the expression of TLR4 in dendritic cells via miR-203. Cell Immunol. 2014;292(1-2):65-9.

22. Chevillet JR, Kang Q, Ruf IK, Briggs HA, Vojtech LN, Hughes SM, et al. Quantitative and stoichiometric analysis of the microRNA content of exosomes. Proc Natl Acad Sci USA. 2014;111(41):14888-93.

23. Haneklaus M, Gerlic M, O'Neill LA, Masters SL. miR-223: infection, inflammation and cancer. J Intern Med. 2013;274(3):215-26.

24. Josse J, Velard F, Mechiche Alami S, Brun V, Guillaume C, Kerdjoudj H, et al. Increased internalization of Staphylococcus aureus and cytokine expression in human Wharton's jelly mesenchymal stem cells. Biomed Mater Eng. 2014;24(1 Suppl):27-35.

25. Yu DS, Wang YS, Bi YL, Guo ZP, Yuan YJ, Tong SM, et al. Salvianolic acid A ameliorates the integrity of blood-spinal cord barrier via miR-101/Cul3/ Nrf2/HO-1 signaling pathway. Brain Res. 2017;1657:279-87.

26. Zhou W, Fong MY, Min Y, Somlo G, Liu L, Palomares MR, et al. Cancersecreted miR-105 destroys vascular endothelial barriers to promote metastasis. Cancer Cell. 2014;25(4):501-15.

27. Zhao L, Wang P, Liu Y, Ma J, Xue Y. miR-34c regulates the permeability of blood-tumor barrier via MAZ-mediated expression changes of ZO-1, occludin, and claudin-5. J Cell Physiol. 2015;230(3):716-31.

28. Ma J, Yao Y, Wang P, Liu Y, Zhao L, Li Z, Li Z, Xue Y. MiR-181a regulates blood-tumor barrier permeability by targeting Kruppel-like factor 6. J Cereb Blood Flow Metab. 2014;34:1826-36.

29. Bischoff SC, Kramer S. Human mast cells, bacteria, and intestinal immunity. Immunol Rev. 2007;217:329-37.

30. Ravanbakhsh N, Kesavan A. The role of mast cells in pediatric gastrointestinal disease. Ann Gastroenterol. 2019;32(4):338-45.

31. De Zuani M, Dal Secco C, Frossi B. Mast cells at the crossroads of microbiota and IBD. Eur J Immunol. 2018:48(12):1929-37.

32. Wilcock A, Bahri R, Bulfone-Paus S, Arkwright PD. Mast cell disorders: from infancy to maturity. Allergy. 2019;74(1):53-63.

33. Lennon EM, Borst LB, Edwards LL, Moeser AJ. Mast cells exert anti-inflammatory effects in an IL $10(-/-)$ model of spontaneous colitis. Mediators Inflamm. 2018;2018:7817360.

34. Kannen V, Sakita JY, Carneiro ZA, Bader M, Alenina N, Teixeira RR, et al. Mast cells and serotonin synthesis modulate chagas disease in the colon: clinical and experimental evidence. Dig Dis Sci. 2018;63(6):1473-84.

35. Wilcz-Villega EM, McClean S, O'Sullivan MA. Mast cell tryptase reduces junctional adhesion molecule-A (JAM-A) expression in intestinal epithelial cells: implications for the mechanisms of barrier dysfunction in irritable bowel syndrome. Am J Gastroenterol. 2013;108(7):1140-51.

36. Mathivanan $\mathrm{S}$, Ji H, Simpson RJ. Exosomes: extracellular organelles important in intercellular communication. J Proteomics. 2010;73(10):1907-20.

37. Valadi H, Ekstrom K, Bossios A, Sjostrand M, Lee JJ, Lotvall JO. Exosomemediated transfer of mRNAs and microRNAs is a novel mechanism of genetic exchange between cells. Nat Cell Biol. 2007:9(6):654-9.

38. Al-Nedawi K, Szemraj J, Cierniewski CS. Mast cell-derived exosomes activate endothelial cells to secrete plasminogen activator inhibitor type 1. Arterioscler Thromb Vasc Biol. 2005;25(8):1744-9.

39. Zhang L, Zhang F, He DK, Fan XM, Shen J. MicroRNA-21 is upregulated during intestinal barrier dysfunction induced by ischemia reperfusion. Kaohsiung J Med Sci. 2018;34:556-63.

40. Hsu YL, Hung JY, Chang WA, Lin YS, Pan YC, Tsai PH, Wu CY, Kuo PL. Hypoxic lung cancer-secreted exosomal miR-23a increased angiogenesis and vascular permeability by targeting prolyl hydroxylase and tight junction protein ZO-1. Oncogene. 2017;36:4929-42.

41. Burek M, Konig A, Lang M, Fiedler J, Oerter S, Roewer N, Bohnert M, Thal SC, Blecharz-Lang KG, Woitzik J, Thum T, Forster CY. Hypoxia-induced MicroRNA-212/132 alter blood-brain barrier integrity through inhibition of tight junction-associated proteins in human and mouse brain microvascular endothelial cells. Transl Stroke Res. 2019:10:672-83.

42. Tang Y, Zhang L, Forsyth CB, Shaikh M, Song S, Keshavarzian A. The role of miR-212 and iNOS in alcohol-induced intestinal barrier dysfunction and steatohepatitis. Alcohol Clin Exp Res. 2015;39:1632-41.
43. Ge X, Han Z, Chen F, Wang H, Zhang B, Jiang R, Lei P, Zhang J. MiR-21 alleviates secondary blood-brain barrier damage after traumatic brain injury in rats. Brain Res. 2015;1603:150-7.

44. Ge X, Huang S, Gao H, Han Z, Chen F, Zhang S, Wang Z, Kang C, Jiang R, Yue S, Lei P, Zhang J. miR-21-5p alleviates leakage of injured brain microvascular endothelial barrier in vitro through suppressing inflammation and apoptosis. Brain Res. 2016;1650:31-40.

45. Zhang L, Shen J, Cheng J, Fan X. MicroRNA-21 regulates intestinal epithelial tight junction permeability. Cell Biochem Funct. 2015;33:235-40.

46. Zhu Y, Wang W, Yuan T, Fu L, Zhou L, Lin G, Zhao S, Zhou H, Wu G, Wang J. MicroRNA-29a mediates the impairment of intestinal epithelial integrity induced by intrauterine growth restriction in pig. Am J Physiol Gastrointest Liver Physiol. 2017;312:G434-42.

47. Martinez C, Rodino-Janeiro BK, Lobo B, Stanifer ML, Klaus B, Granzow M, Gonzalez-Castro AM, Salvo-Romero E, Alonso-Cotoner C, Pigrau M, Roeth R, Rappold G, Huber W, Gonzalez-Silos R, Lorenzo J, de Torres I, Azpiroz F, Boulant S, Vicario M, Niesler B, Santos J. miR-16 and miR-125b are involved in barrier function dysregulation through the modulation of claudin-2 and cingulin expression in the jejunum in IBS with diarrhoea. Gut. 2017:66:1537-8.

48. Ye EA, Liu L, Steinle JJ. miR-15a/16 inhibits TGF-beta3/NEGF signaling and increases retinal endothelial cell barrier proteins. Vision Res. 2017:139:23-9.

49. Miao YS, Zhao YY, Zhao LN, Wang P, Liu YH, Ma J, Xue YX. MiR-18a increased the permeability of BTB via RUNX1 mediated down-regulation of ZO-1, occludin and claudin-5. Cell Signal. 2015;27:156-67.

50. Zhao YY, Zhao LN, Wang P, Miao YS, Liu YH, Wang ZH, Ma J, Li Z, Li ZQ, Xue $Y X$. Overexpression of miR-18a negatively regulates myocyte enhancer factor 2D to increase the permeability of the blood-tumor barrier via Kruppel-like factor 4-mediated downregulation of zonula occluden-1, claudin-5, and occludin. J Neurosci Res. 2015:93:1891-902.

51. Miyata R, Kakuki T, Nomura K, Ohkuni T, Ogasawara N, Takano K, Konno T, Kohno T, Sawada N, Himi T, Kojima T. Poly(l:C) induced microRNA-146a regulates epithelial barrier and secretion of proinflammatory cytokines in human nasal epithelial cells. Eur J Pharmacol. 2015;761:375-82.

52. Cordes F, Bruckner M, Lenz P, Veltman K, Glauben R, Siegmund B, Hengst K, Schmidt MA, Cichon C, Bettenworth D. MicroRNA-320a strengthens intestinal barrier function and follows the course of experimental colitis. Inflamm Bowel Dis. 2016;22:2341-55.

53. Chen T, Xue H, Lin R, Huang Z. MiR-34c and PIncRNA1 mediated the function of intestinal epithelial barrier by regulating tight junction proteins in inflammatory bowel disease. Biochem Biophys Res Commun. 2017:486:6-13

54. Wang L, Zhang R, Chen J, Wu Q, Kuang Z. Baicalin protects against TNFalpha-induced injury by down-regulating miR-191a that targets the tight junction protein ZO-1 in IEC-6 cells. Biol Pharm Bull. 2017;40:435-43.

55. Yang J, Liu X, Yuan X, Wang Z. miR-99b promotes metastasis of hepatocelIular carcinoma through inhibition of claudin 11 expression and may serve as a prognostic marker. Oncol Rep. 2015;34:1415-23.

56. Liu Z, Tian Y, Jiang Y, Chen S, Liu T, Moyer MP, Qin H, Zhou X. Protective effects of Let-7b on the expression of occludin by targeting P38 MAPK in preventing intestinal barrier dysfunction. Cell Physiol Biochem. 2018:45:343-55.

57. Zhao H, Zhao C, Dong Y, Zhang M, Wang Y, Li F, Li X, McClain C, Yang S, Feng W. Inhibition of miR122a by Lactobacillus rhamnosus GG culture supernatant increases intestinal occludin expression and protects mice from alcoholic liver disease. Toxicol Lett. 2015;234:194-200.

58. Zhu M, Fei L, Li D, Chen D. Correlation analysis of miR-122-5p and occludin with sperm density in oligospermia patients'sperm. Clin Lab. 2019. https://doi.org/10.7754/Clin.Lab.2018.180814.

59. Zuo X, Lu J, Manaenko A, Qi X, Tang J, Mei Q, Xia Y, Hu Q. MicroRNA-132 attenuates cerebral injury by protecting blood-brain-barrier in MCAO mice. Exp Neurol. 2019;316:12-9.

60. Avalle L, Incarnato D, Savino A, Gai M, Marino F, Pensa S, Barbieri I, Stadler MB, Provero P, Oliviero S, Poli V. MicroRNAs-143 and -145 induce epithelial to mesenchymal transition and modulate the expression of junction proteins. Cell Death Differ. 2017;24:1750-60.

61. Bai Y, Zhang Y, Hua J, Yang X, Zhang X, Duan M, Zhu X, Huang W, Chao J, Zhou R, Hu G, Yao H. Silencing microRNA-143 protects the integrity of the blood-brain barrier: implications for methamphetamine abuse. Sci Rep. 2016;6:35642. 
62. Mishra R, Singh SK. HIV-1 Tat C modulates expression of miRNA-101 to suppress VE-cadherin in human brain microvascular endothelial cells. J Neurosci. 2013;33:5992-6000.

63. Ge X, Li W, Huang S, Yin Z, Yang M, Han Z, Han Z, Chen F, Wang H, Lei $P$, Zhang J. Increased miR-21-3p in injured brain microvascular endothelia cells after traumatic brain injury aggravates blood-brain barrier damage by promoting cellular apoptosis and inflammation through targeting MAT2B. J Neurotrauma. 2019;36:1291-305.

64. Ma Q, Dasgupta C, Li Y, Huang L, Zhang L. MicroRNA-210 suppresses junction proteins and disrupts blood-brain barrier integrity in neonatal rat hypoxic-ischemic brain injury. Int J Mol Sci. 2017. https://doi.org/10.3390/ ijms18071356.

65. Ma T, Xue YX. MiRNA-200b regulates RMP7-induced increases in bloodtumor barrier permeability by targeting RhoA and ROCKII. Front Mol Neurosci. 2016;9:9.

66. Shen Y, Zhou M, Yan J, Gong Z, Xiao Y, Zhang C, Du P, Chen Y. miR-200b inhibits TNF-alpha-induced IL-8 secretion and tight junction disruption of intestinal epithelial cells in vitro. Am J Physiol Gastrointest Liver Physiol. 2017;312:G123-32.

67. Zhao W, Wang P, Ma J, Liu YH, Li Z, Li ZQ, Wang ZH, Chen LY, Xue YX. MiR34 a regulates blood-tumor barrier function by targeting protein kinase Cepsilon. Mol Biol Cell. 2015;26:1786-96.

68. Zhang H, Zhang S, Zhang J, Liu D, Wei J, Fang W, Zhao W, Chen Y, Shang D. ZO-1 expression is suppressed by GM-CSF via miR-96/ERG in brain microvascular endothelial cells. J Cereb Blood Flow Metab. 2018;38:809-22.
69. Fang Z, He QW, Li Q, Chen XL, Baral S, Jin HJ, Zhu YY, Li M, Xia YP, Mao $L$, Hu B. MicroRNA-150 regulates blood-brain barrier permeability via Tie-2 after permanent middle cerebral artery occlusion in rats. FASEB J. 2016;30:2097-107.

70. Yu T, Lu XJ, Li JY, Shan TD, Huang CZ, Ouyang H, Yang HS, Xu JH, Zhong W, Xia ZS, Chen QK. Overexpression of miR-429 impairs intestinal barrier function in diabetic mice by down-regulating occludin expression. Cell Tissue Res. 2016;366:341-52.

71. Liu W, Cai H, Lin M, Zhu L, Gao L, Zhong R, Bi S, Xue Y, Shang X. MicroRNA-107 prevents amyloid-beta induced blood-brain barrier disruption and endothelial cell dysfunction by targeting Endophilin-1. Exp Cell Res. 2016:343:248-57.

72. Chang CW, Yu JC, Hsieh YH, Yao CC, Chao JI, Chen PM, Hsieh HY, Hsiung CN, Chu HW, Shen CY, Cheng CW. MicroRNA-30a increases tight junction protein expression to suppress the epithelial-mesenchymal transition and metastasis by targeting Slug in breast cancer. Oncotarget. 2016;7:16462-78

73. Cai H, Xue Y, Wang P, Wang Z, Li Z, Hu Y, Li Z, Shang X, Liu Y. The long noncoding RNA TUG1 regulates blood-tumor barrier permeability by targeting miR-144. Oncotarget. 2015;6:19759-79.

\section{Publisher's Note}

Springer Nature remains neutral with regard to jurisdictional claims in published maps and institutional affiliations.
Ready to submit your research? Choose BMC and benefit from:

- fast, convenient online submission

- thorough peer review by experienced researchers in your field

- rapid publication on acceptance

- support for research data, including large and complex data types

- gold Open Access which fosters wider collaboration and increased citations

- maximum visibility for your research: over $100 \mathrm{M}$ website views per year

At BMC, research is always in progress.

Learn more biomedcentral.com/submissions 